\section{§14. Linear Polarization of Photon Emissions from Reflected Neutrals of Atomic Hydrogen at High-Z First Wall Surfaces}

Kato, D.,

Kenmotsu, T. (Doshisha Univ.),

Ohya, K. (Tokushima Univ.),

Tanabe, T. (Kyushu Univ.)

Spectroscopic studies identify Doppler-shifted Balmer-line emission from super-thermal hydrogen atoms reflected at high- $Z$ metal surfaces exposed to hydrogen plasmas. The reflection becomes more important in fuel recycling at the high- $Z$ first-walls, because of its larger reflection coefficients. The line intensity and shape have been analyzed taking account of collisions with the plasma particles. In the analysis, it has been presumed that the reflected neutrals were initially in the ground state. This naive assumption has been widely adopted, since excited state population in the reflected neutrals was unknown.

Experimental studies of photon emission from excited states in reflected neutrals have been reported for high-Z first-wall materials. However, few measurements are found for the polarization in literatures. One previous measurement is by Winter and Hagedorn for Lyman- $\alpha$ from hydrogen atoms specularly reflected at grazing angles on a $\mathrm{Ni}(111)$ surface. In the present work, the linear polarizations of $2 p-1 s$ and $3 d-2 p$ line emissions from hydrogen atoms created by electron capture above $\mathrm{W}$ surfaces are investigated theoretically. The linear polarization degrees are evaluated assuming that photons are detected at 90 degree to the surface normal.

In the present work ${ }^{1-2)}$, it is assumed that the excited states are formed via single electron capture by a hydrogen nucleus from conduction bands. The electron transfer is described by using semi-classical theories (electronic degrees of freedom are treated quantum mechanically, while nucleus motion is represented by classical trajectories). In the present calculations, for the sake of simplicity, hydrogen nuclei recede from metal surfaces along straight-line classical trajectories of constant velocities parallel to the surface normal, i.e. along $\mathrm{z}$-axis.

Contour plots for electron densities of excited states above $\mathrm{W}$ surface are shown in Fig. 1. The plots are presented for the two ion velocities, 0.2 and $0.8 \mathrm{au}$. The densities are asymmetric along $z$-axis indicating hybridization of the sub-shell orbital. For the smaller velocity, the electron density is stretched to far side from the surface, since the resonance ionization preferentially depopulates electrons near the surface. Contrary to this, for the larger velocity, the density is stretched toward the surface side. At high velocities, net interaction between hydrogen nucleus and surfaces becomes weaker and described with perturbation theories. In the perturbation theories, electron capture probabilities are in proportion to overlap between wavefunctions of atomic and surface electrons. Since hybridized atomic orbital stretched toward to the surface has larger overlap, giving lager probabilities for the electron capture. It is noted that the resonance ionization is also enhanced as the overlap becomes larger, however that is of the second or higher order processes in the perturbation series which are negligible in the weak interaction. Fig. 2 shows linear polarization degrees of $2 p-$ $1 \mathrm{~s}$ and $3 \mathrm{~d}-2 \mathrm{p}$ lines as a function of the ion velocity. Negative polarization is obtained in this velocity range. As the ion velocity increases, however, degrees of the negative polarization become smaller. Reflection would produce outgoing particles with velocities distributed in a wide rage. Apparent linear polarization degrees are, therefore, obtained by averaging with the velocity distribution of photon emitting particles.

This work is supported by KAKENHI(19055005).

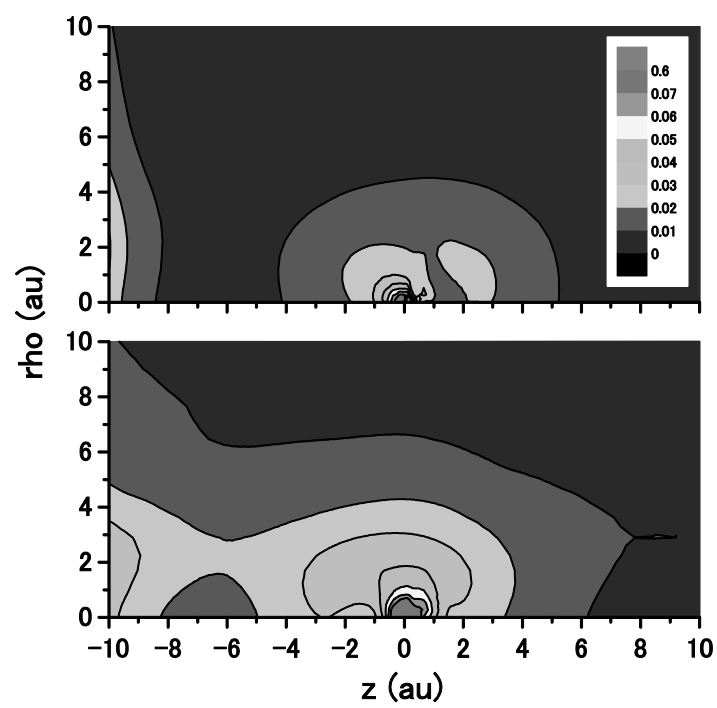

Fig.1 Contour plot of electron density of excited states created by electron capture from $\mathrm{W}$ surface in the cylindrical coordinates. Above: ion velocity is 0.2 au, below: 0.8 au. W surface edge is located at $z=-11$ au (outside of this figure). Electron density of $1 \mathrm{~s}$ orbital is excluded. The electron density is unchanged by rotation along $z$-axis.

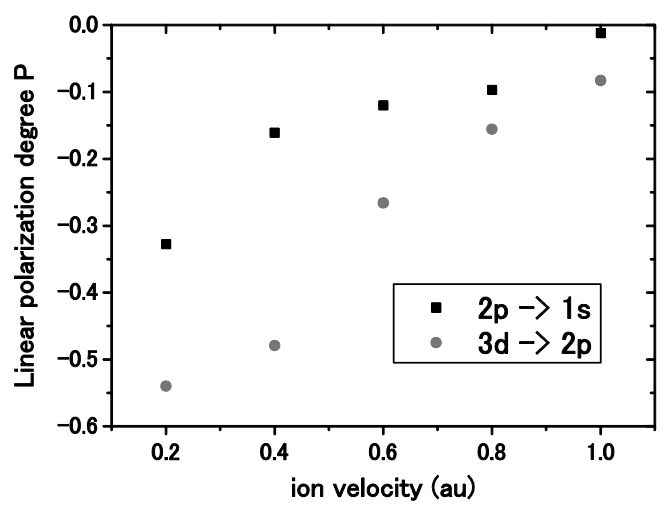

Fig.2 Linear polarization degrees of $2 p-1 s$ and $3 d-2 p$ lines as a function of ion velocity.

1) Kato, D. et al., Contributions to Plasma Physics 50 (2010) 445.

2) Kato, D. et al., J. Nucl. Mater, accepted (2011). 